\section{Translationale (Laboratoriums-) Medizin}

\author{
A. M. Gressner ${ }^{1}$ und O. A. Gressner ${ }^{2}$ \\ ${ }^{1}$ Labor Dr. Wisplinghoff Berlin, Berlin, Deutschland \\ ${ }^{2}$ Labor Dr. Wisplinghoff Köln, Köln, Deutschland
}

Synonym(e) Translationale Forschung

Englischer Begriff translational (laboratory-) medicine; translational research

Definition Translationale Medizin (TM) überführt neue (Grundlagen-) wissenschaftliche Erkenntnisse in einem geordneten Verfahren (z. B. klinische Studien) zur klinischen Anwendung in Diagnose, Therapie und Prävention spezifischer Erkrankungen. TM entwickelt integrative Konzepte, denen zufolge eine strategisch geplante, rasche und konsequente Umsetzung der Grundlagenforschung in die klinische Realität ermöglicht wird.

Beschreibung Zielsetzung der translationalen (Labor-) Medizin ist es, neue Ergebnisse der biomedizinischen Grundlagen- oder Patienten-orientierten Forschung (z. B. Stammzell- und Genomforschung) sowie technologische Inno- vationen (z. B. Nanotechnologie, \ Micro-RNA) für eine Verbesserung der medizinischen Patientenversorgung konsequent und zielgerichtet nutzbar zu machen. Dazu gehören auf labordiagnostischem Sektor beispielsweise Früherkennungsparameter von Tumoren, Infektionen, Entzündungen und ihrer Komplikationen, Krankheitsprädiktoren (z. B. neurodegenerativer und kardiovaskulärer Erkrankungen), innovative Prognoseparameter und Methoden des kontinuierlichen In-situ-Monitorings von Laborparametern bei Intensivpatienten. TM betreibt konsequent und rasch die Bench-to-bedside-Forschung und -Entwicklung in einem geordneten, strategischen Evaluationsverfahren (z. B. experimentelle und humane diagnostische oder therapeutische Studien) an der Nahtstelle zwischen wissenschaftlicher Entdeckung und ärztlichem Erfordernis. TM ist für forschungsaktive Universitäten, die innovative Industrie und außeruniversitäre Forschungseinrichtungen weltweit $\mathrm{zu}$ einem zentralen Kooperationsschwerpunkt geworden.

\section{Literatur}

Hörig H, Marincola E, Marincola MF (2005) Obstacles and opportunities in translational research. Nat Med 11:705-708

Sonntag K-C (2005) Implementations of translational medicine. J Transl Med 3:33-35 